\title{
Application of Modified Rapid Thermal Annealing to Doped Polycrystalline Si Thin Films Towards Low Temperature Si Transistors.
}

\author{
Byung-Soo So, Hyeong-June Kim, Young- Hwan Kim, Jin-Ha Hwang ${ }^{\dagger}$ \\ Department of Materials Science \& Engineering, Hongik University, Seoul 121-791
}

(Received August 6, 2008 : Revised October 10, 2008 : Acceped October 13, 2008)

\begin{abstract}
Modified thermal annealing was applied to the activation of the polycrystalline silicon films doped as p-type through implantation of $\mathrm{B}_{2} \mathrm{H}_{6}$. The statistical design of experiments was successfully employed to investigate the effect of rapid thermal annealing on activation of polycrystalline Si doped as p-type. In this design, the input variables are furnace temperature, power of halogen lamps, and alternating magnetic field. The degree of ion activation was evaluated as a function of processing variables, using Hall effect measurements and Raman spectroscopy. The main effects were estimated to be furnace temperature and RTA power in increasing conductivity, explained by recrystallization of doped ions and change of an amorphous $\mathrm{Si}$ into a crystalline Si lattice. The ion activation using rapid thermal annealing is proven to be a highly efficient process in low temperature polycrystalline Si technology.
\end{abstract}

Key words ion activation, polycrystalline $\mathrm{Si}$, conductivity, Raman spectroscopy, rapid thermal annealing.

\section{Introduction}

Polycrystalline silicon (poly-Si) thin film transistors (TFT's) on glass substrates have been recognized as essential components in active matrix organic lightemitting diodes (AMOLED's). Low temperature polycrystalline Si (LTPS) TFT's have opened an innovative area for the next generation of flat-panel displays, due to higher mobility of charge carriers, relative to amorphous $\mathrm{Si}$ (a-Si) TFT's. LTPS transistors offer $10 \sim 500 \mathrm{~cm}^{2} / \mathrm{V} \cdot \mathrm{sec}$ than those of the a-Si TFT ranging from $0.3 \sim 1.0 \mathrm{~cm}^{2} / \mathrm{V} \cdot \mathrm{sec}^{1-5)}$ Higher mobility offers i) size reduction in transistors leading to higher aperture ratio, ii) economical manufacturing cost through the incorporation of the integration of driver ICs into glass substrates, and iii) less weight and thickness in display modules. In addition, LTPS transistors possess high potential towards multifunctional systems-on-glasses, including the panel ASIC, memories, photodiodes, functional sensors, sound chips, etc. The unique advantages of polycrystalline silicon TFT provide an ideal backbone for AMOLED displays whose characteristics possess excellent color coordinates, selfemitting feature, fast on/off operations, wide viewing angle, etc. However, the AMOLED's require a strict control

Corresponding author E-Mail : jhwang@hongik.ac.kr (J. H. Hwang) in TFT characteristics with emphasis on Si crystallization, gate dielectrics, and ion-doping. In particular, the LTPS technology has been advanced, based on various crystallization techniques: e.g., sold phase crystallization, excimer laser annealing, rapid thermal annealing, metalinduced crystallization, etc,. ${ }^{6-14)}$ The crystallization techniques can be adapted for activation of ion-doped Si thin films, by making the possibility through slight modifications in processing variables.

Ion-doping process accompanies the activation of ions implanted into polycrystalline $\mathrm{Si}$ (p-Si) as n-type or p-type. Depending on type of ion-doping, condition of activation, and dose amount, the resultant transistor characteristics vary significantly in transistor performance. Ions are doped into the polycrystalline Si lattice in form of either implanted ions such as $\mathrm{B}+$ and $\mathrm{P}+$ or charged ions dissociated from of $\mathrm{B}_{2} \mathrm{H}_{6}$ and $\mathrm{PH}_{3}$. The detailed activation processes are furnace annealing, rapid thermal annealing, excimer laser annealing, etc. ${ }^{15-18)}$ In conventional furnace annealing, the processing is performed at 500 to $600^{\circ} \mathrm{C}$. Rapid thermal annealing (RTA) is processed around or below $1000^{\circ} \mathrm{C}$ in nitrogen. Rapid thermal annealing and excimer laser annealing is proven to be more efficient than thermal annealing in terms of time and thermal budget in the LTPS process maps.

We combined the rapid thermal annealing with infrared heating and magnetic field in order to provide highly 
conductive Si thin films, which allows the robust source/ drain electrodes. The current report will address the performance based on processing variables using statistical design of experiments. For highly effective thin film transistors, the source and drain electrodes must possess low sheet resistance which requires higher degree of activation. Therefore, the conductivity is chosen as an output parameter. The work reports the application of a statistical approach based on design of experiments to ion activation which incorporates infrared heating and magnetic field with emphasis on rapid heat treatments.

\section{Experimental procedure}

The activation process was performed using the rapid thermal annealing system as shown in Fig. 1. The frequency of alternating current is $200 \mathrm{kHz}$ and alternating current ranges up to $20 \mathrm{~A}$. When alternating current is applied through induction coils around halogen lamps, it creates magnetic field and changes the direction of induced magnetic fields, as a function of the initial ac electric field. Adjacent to the main processing module, the additional furnaces function as a heating and cooling

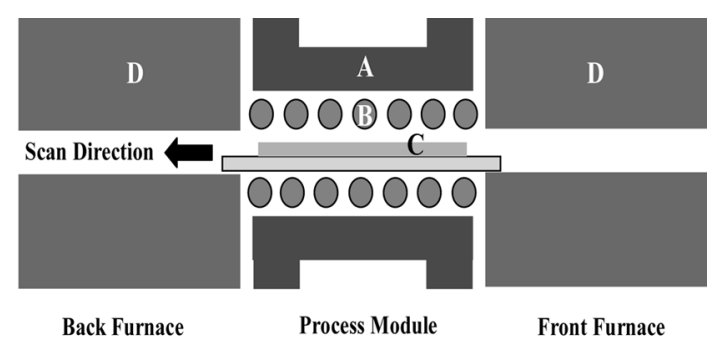

Fig. 1. Schematic diagram of rapid thermal annealing process module (A: magnet coil part, B: halogen lamp part, C: Si/Corning 1737 substrate on quartz tray, and D: heating body). system in order to avoid the undesired warping or mechanical damage. The scanning rate of glass substrates was $14 \mathrm{~mm} / \mathrm{sec}$ and nitrogen gas was flowed in the process module to avoid degradation of $\mathrm{Si}$ thin film during the activation process (about $3 \mathrm{l} / \mathrm{min}$ ).

Ion activation is performed as a function of furnace temperature, magnetic power, and halogen lamp power (RTA power). The full factorial $\left(2^{3}\right)$ design created 8 different runs of the high and low value (denoted by +1 and -1 in coded values, respectively) in experiment variables with three center points. The detailed values are shown in Table 1. Finally, the eleven runs was performed in this design 11 as shown in Table 1 because we added three center points which can test the reproducibility in experimental runs. The standard order was randomized in order to yield the actual run order and eliminate the timedependent influence that is not a controllable factor.

Amorphous $\mathrm{Si}$ was deposited on the $\mathrm{SiO}_{2}(3000 \AA) /$ glass (Corning 1737) at the thickness of $500 \AA$ by plasmaenhanced chemical vapor deposition (PECVD). Subsequently the amorphous $\mathrm{Si}$ was crystallized through excimer laser annealing (wavelength of $308 \mathrm{~nm}$ with $\mathrm{XeCl}$ source). Ion-doping was conducted as p-type on the polycrystalline $\mathrm{Si}$ supported on glass substrates through ion shower doping of $\mathrm{B}_{2} \mathrm{H}_{6}$ : the doped species includes ionized species dissociated from $\mathrm{B}_{2} \mathrm{H}_{6}$, i.e., $\mathrm{B}_{2} \mathrm{H}_{5}^{+1}, \mathrm{~B}_{2} \mathrm{H}_{4}^{+2}$, $\mathrm{B}_{2} \mathrm{H}_{3}{ }^{+3}$, etc. The specimens were cut into $10 \mathrm{~mm} \times 10 \mathrm{~mm}$ plates for activation test and electrical characterization. The electrical properties were measured using Hall measurements through a Van der Pauw configuration. The ohmic contact between electrodes and doped regions was confirmed, using a dc (direct current) four-point resistivity measurement technique on the identical specimens indicating that there is no nonohmic contribution at the

Table 1. The processing conditions obtained using the design of experiments and the corresponding electrical data

\begin{tabular}{cccccccc}
\hline Sta. order & Run Order & $\begin{array}{c}\text { Furnace Temp. } \\
{\left[{ }^{\circ} \mathrm{C}\right]}\end{array}$ & $\begin{array}{c}\text { RTA Power } \\
{[\%]}\end{array}$ & $\begin{array}{c}\text { Magnetic Power } \\
{[\mathrm{A}]}\end{array}$ & $\begin{array}{c}\text { Conductivity } \\
\left(\Omega^{-1} \mathrm{~cm}^{-1}\right)\end{array}$ & $\begin{array}{c}\text { Mobility } \\
\left(\mathrm{cm}^{2} / \mathrm{V}-\mathrm{s}\right)\end{array}$ & $\begin{array}{c}\text { Carrier Conc. } \\
\left(\times 10^{19} \mathrm{~cm}^{-3}\right)\end{array}$ \\
\hline 3 & 1 & $640(-1)$ & $40(+1)$ & $0(-1)$ & 63 & 32.3 & 1.22 \\
1 & 2 & $640(-1)$ & $10(-1)$ & $0(-1)$ & 59 & 28.3 & 1.29 \\
11 & 3 & $670(0)$ & $25(0)$ & $10(0)$ & 66 & 29.1 & 1.46 \\
10 & 4 & $670(0)$ & $25(0)$ & $10(0)$ & 64 & 29.8 & 1.34 \\
2 & 5 & $700(+1)$ & $10(-1)$ & $0(-1)$ & 71 & 70.8 & 0.63 \\
5 & 6 & $640(-1)$ & $10(-1)$ & $20(+1)$ & 58 & 32.4 & 1.11 \\
6 & 7 & $700(+1)$ & $10(-1)$ & $20(+1)$ & 69 & 84.5 & 0.51 \\
9 & 8 & $670(0)$ & $25(0)$ & $10(0)$ & 63 & 32.4 & 1.22 \\
7 & 9 & $640(-1)$ & $40(+1)$ & $20(+1)$ & 69 & 31.3 & 1.36 \\
4 & 10 & $700(+1)$ & $40(+1)$ & $0(-1)$ & 82 & 34.7 & 1.48 \\
8 & 11 & $700(+1)$ & $40(+1)$ & $20(+1)$ & 78 & 34.4 & 1.41 \\
\hline
\end{tabular}


electrode/semiconductor interface. During Hall Effect measurements the four microtips were placed on the corners of the squared specimens to eliminate the stray contributions resulting from poor electroding. Sheet resistance was calculated by taking into account geometric factors. Hall measurements confirmed the type of charge carriers.

\section{Results and Discussion}

Design of experiments allows estimation of importance of primary effects and interactions and modeling of the process under study, i.e., the activation in ion-doping. The system can be modeled as the following

$$
\begin{aligned}
Y=\beta_{0}+\beta_{1} x_{1}+ & \beta_{2} x_{2}+\beta_{3} x_{3}+\beta_{12} x_{1} x_{2}+ \\
\beta_{13} x_{1} x_{3}+ & \beta_{23} x_{2} x_{3}+\beta_{123} x_{1} x_{2} x_{3}+\varepsilon
\end{aligned}
$$

where $Y$ is the output variable (i.e., conductivity), $\mathrm{x}_{1}$ represents factor $\mathrm{A}, \mathrm{x}_{2}$ represents factor $\mathrm{B}, \mathrm{x}_{1} \mathrm{x}_{2}$ represents the two-factor interaction, specifically the $\mathrm{AB}$ interaction, and $\mathrm{x}_{1} \mathrm{x}_{2} \mathrm{x}_{3}$ represents the $\mathrm{ABC}$ interaction. $\beta_{0}$ is the grand average of the observations, and $\beta_{1}, \beta_{2}, \beta_{12}, \beta_{123}$, etc are estimated by one-half of corresponding effect values and $\varepsilon$ is a random error. ${ }^{15)}$ Fortunately, the current work was performed through a full-factorial design with 3 center points; three factors with two levels, leading to the total of 11 experiments. The three center points are employed to check the reproducibility in DOE. In this work, the center points are 3,4, and 8 in the run order specified in Table 1 . The reproducibility is extremely excellent, e.g., $63 \sim 64 \Omega^{-1} \mathrm{~cm}^{-1}$. The detailed information is summarized in Table 2 where effects and coefficients of Eq.(1) are shown. As can be seen in Table 2, two main factors indicate considerably high value of t-values, larger than 3. No multi-factor interactions are analyzed to be statistical significant, meaning that the corresponding factors should be taken into account in activation processing. From Eq. (1) and Table 2, coded mathematical models are simplified.

$$
\text { Conductivity }(p \text {-type })=68.5+6.6 x_{1}+4.3 x_{2}
$$

Based on the reproducibility, the detailed analysis are shown in the normal plot (Fig. 2(a)) and Pareto chart (Fig. 2(b)). Table 2 exhibits the experimental results on conductivity obtained for estimating the effect and interactions of the experimental variables in rapid thermal annealing. The current work is analyzed in terms of
Table 2. Estimated effects, coefficients, t-values for sheet resistance in activation

\begin{tabular}{cccc}
\hline Standard order & Effects & Coefficient & t- value \\
\hline Constant & - & 68.5 & 127.8 \\
A & 13.1 & 6.6 & 12.3 \\
B & 8.5 & 4.3 & 7.9 \\
C & -0.4 & -0.2 & -0.4 \\
AB & 1.2 & 0.6 & 1.1 \\
AC & -2.7 & -1.3 & -2.5 \\
BC & 0.7 & 0.4 & 0.7 \\
ABC & -2.4 & -1.2 & -2.2 \\
Center point. & - & -3.9 & -3.8 \\
\hline
\end{tabular}

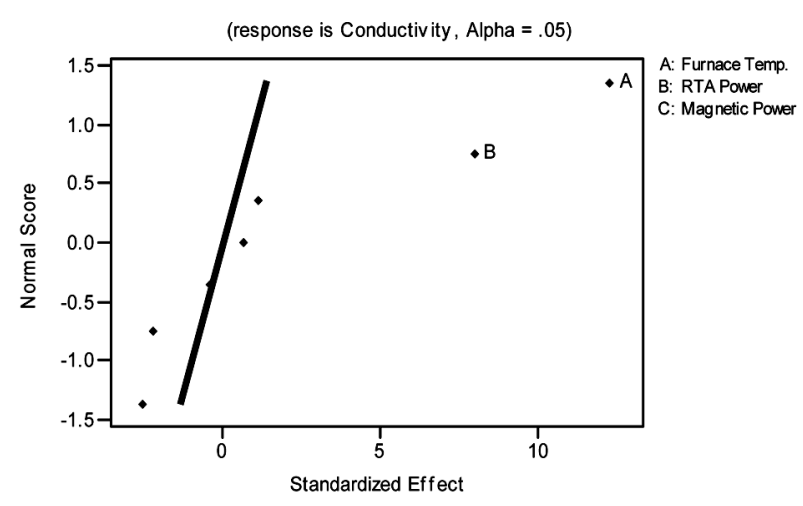

(a)

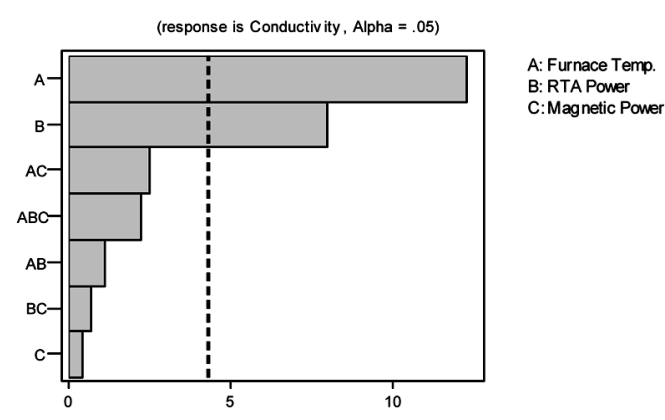

(b)

Fig. 2. (a) Normal probability plot and (b) Pareto chart of conductivity obtained in ion activation using rapid thermal annealing.

applicability of the statistical approach, only considering the absolute magnitude of estimated effects. Since the current design dose not employ fractional factorial design, all main effects and all interactions can be clarified. This will help understanding the main effects and the interactions without ambiguity. Typically, threefactor and high-order factor interactions are assumed to be negligible in estimating the effects, leading to 


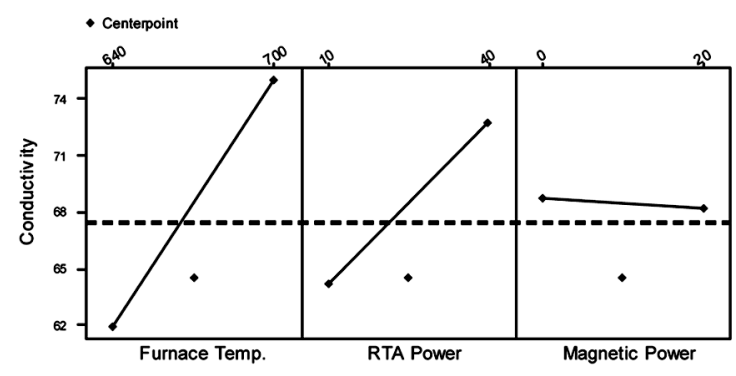

(a)

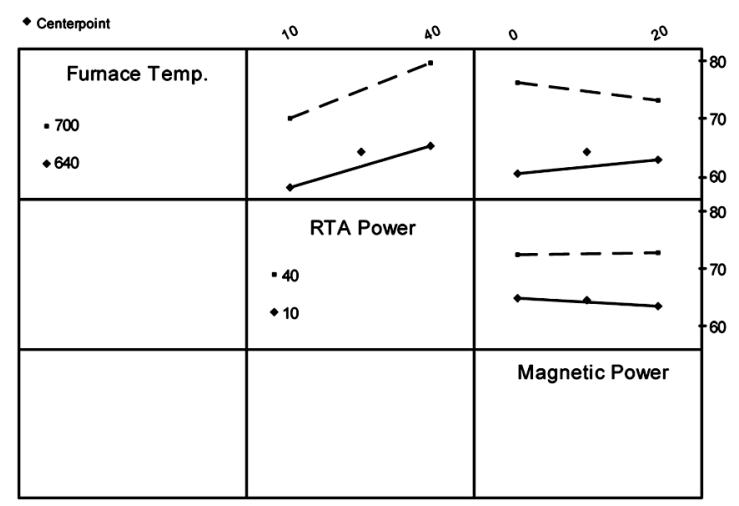

(b)

Fig. 3. (a) Main effects plot and (b) interaction plot in activating doped $\mathrm{Si}$ thin films using modified rapid thermal annealing (Based on experimental results of Table 1).

reduction of experimental effort. The assumption is not applied to the current work under the significance level of 0.05 . In case of p-type activation, only two primary effects, i.e., furnace temperature and RTA power are significant. As shown in Fig. 3(a), the main effects of furnace temperature and RTA power are lager than that of magnetic field during p-type activation. When the magnetic field is applied, the contribution of magnetic field is not detectable. The data reproducibility was estimated to be significantly high, evidenced by high tvalues. The effect of magnetic field will be reported in the more detailed format elsewhere. In terms of the main effects, the furnace temperature is found to be most significant; the conductivity increases with furnace temperature. Secondly, the conductivity increases with increasing RTA power. However, the effect of the RTA power is relatively smaller rather than that of furnace temperature. The weak dependence of magnetic power is reported in this work. From the interaction plot of Fig. 3(b), no significant interaction is found between furnace temperature and RTA power in activating B-doped Si thin films prepared on glass substrates.

In ion-doping polycrystalline $\mathrm{Si}$ thin films in high concentration of boron, high acceleration voltage can induce the structural damage and even the amorphorization in Si thin films, in other words, the displacement of the $\mathrm{Si}$ atoms and the distortion of the crystalline lattice into the amorphous state. The relevant features were investigated using Raman spectroscopy. As shown in Fig. 4, Raman spectra were deconvoluted using a GaussianLorentz distribution in order to quantify the crystalline, amorphous, and defective portions. This damage recovery occurs through curing of defects, diffusion of doping ions and, substitution between $\mathrm{Si}$ atoms and dopants. In this work, Raman spectra are separated by three point GaussianLorenz distribution method in order to achieve accurate fitting. A peak in the vicinity of $517 \mathrm{~cm}^{-1}$ is allocated to the crystalline phase within doped polycrystalline Si thin films. Peaks related to the amorphous phase and defects are appeared at $480 \mathrm{~cm}^{-1}$ and $515 \mathrm{~cm}^{-1}$, respectively. Each proportion was calculated by data-fitting of Raman spectra.

Even the highest degree of activation was achieved as found in Run No. 10, high proportion of a component is found as a defective component, approximately $13 \%$. As the extensive activation progresses, the portion of ionic rearrangement and structural recovery increases

However, the modified rapid thermal annealing cannot eliminate the presence of defective portion which is located between complete amorphous and highly crystalline states. The current work reports the preliminary reports in order to understand the role of rapid thermal annealing in ion activation and to clarify the main contributions to the current activation process, among the experimental variables employed in the current modified rapid thermal annealing. Further work will be reported with aim to understanding the detailed mechanisms in terms of electrical properties and structural components operating in ion-activation associated with rapid thermal annealing

\section{Conclusion}

A $2^{3}$ full factorial design was employed in order to estimate the main effects and interactions in rapid thermal annealing systems integrated with infrared modified rapid heating and magnetic application. The furnace temperature and RTA power affect activation efficiency significantly, 


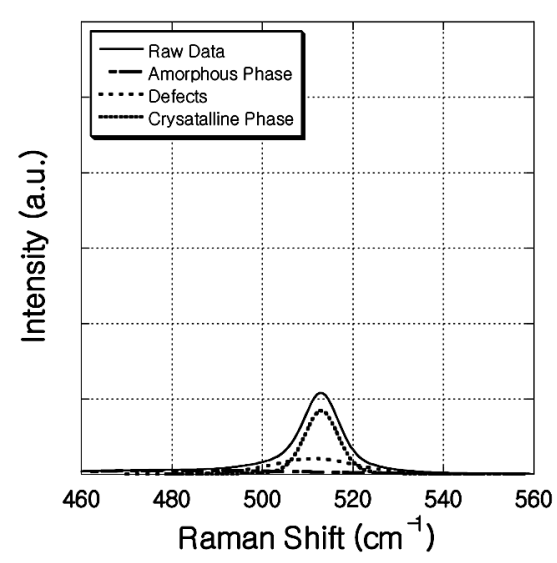

(a)

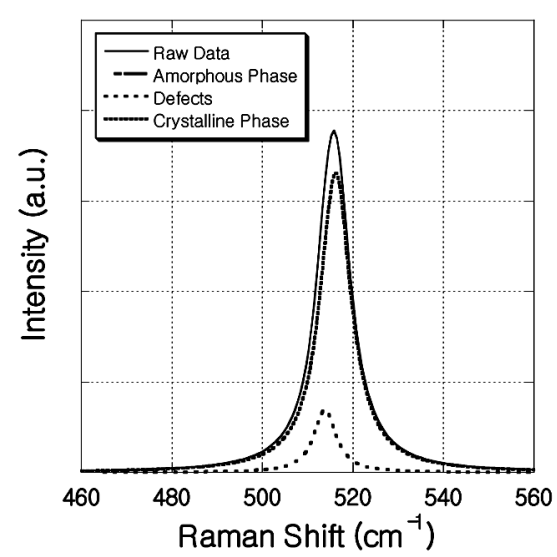

(c)

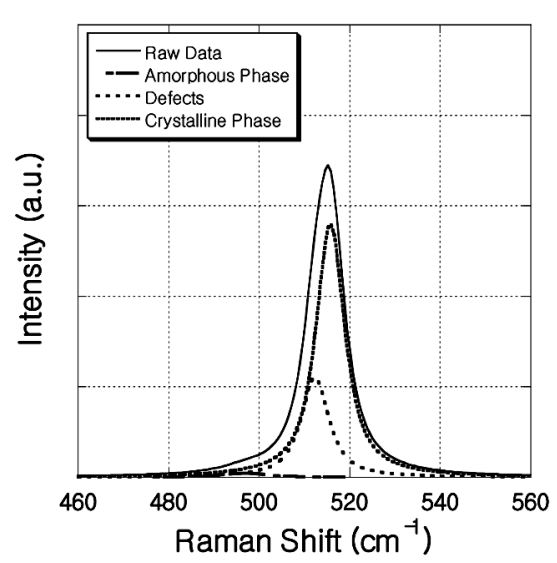

(b)

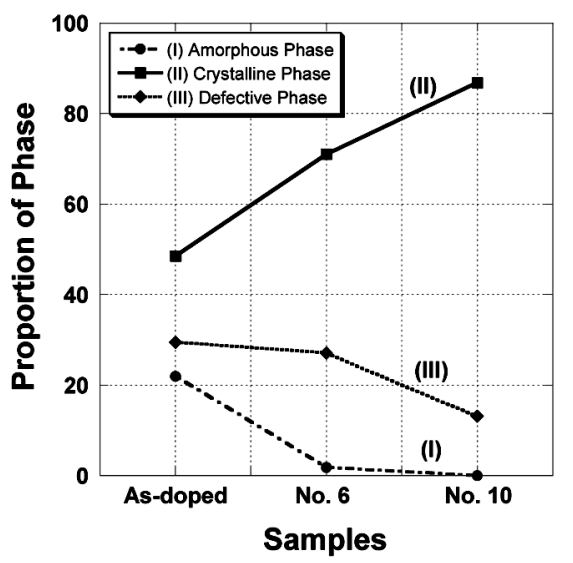

(d)

Fig. 4. Variation of Raman spectra and analyzed proportions of each structural components: (a) after ion doping, (b) after activation of run order No. 6, (c) after activation of run order No. 10, and (d) proportions of each phase.

leading to higher conductivity. The interaction between furnace temperature and RTA power does not have any influence on activation in ion-doping steps in terms of sheet resistance. The high temperature and high infrared heating are confirmed to enhance the conductivity up to $82 \Omega^{-1} \mathrm{~cm}^{-1}$. In particular, it takes less than 15 minutes to activate p-type doped with an ion dose in the order of $10^{14} \mathrm{~cm}^{-2}$. Raman spectra was employed in resolving the structural components originating from the amorphous, defective, and crystalline portions present in the doped polycrystalline Si thn films. In combination with the alternating magnetic field, the current pseudo-rapid thermal processing provides an alternative to the ion activation in low temperature polycrystalline $\mathrm{Si}$ thin film transistors, which recover a structural damage in the LTPS polycrystalline Si induced in ion-doping.

\section{Rerefences}

1. D. Y. He, X. Q.Wang, Q. Chen and J. S. Li, J. Korean Phys. Soc., 46, S88 (2005).

2. N. Komiya, R. Nishikawa, M. Okuyama, T. Yamada, Y. Saito, S. Oima, K. Yoneda, H. Kanno, H. Takahashi, G. Rajeswaran, M. Itoh, M. Boroson and T. K. Hatear, Proceeding of the $10^{\text {th }}$ International Workshop on Inorganic and Organic Electroluminescence, 347 (2000).

3. K. Sera, F. Okumura, H. Uchida, S. Itoh, s. Karelso and K. Hota, IEEE Trans. Electron Device, 36(12), 2868 (1999).

4. M. A. Crowder, P. G. Garey, P. M. Smith, R. S. Sposili, H. S. Cho and J. S. Im, IEEE Electron Device Lett., 19(8), 306 (1998).

5. M. Yamamoto, H. Nishitani, M. Sakai, M. Gotoh, Y. Taketomi, T. Tautsu and M. Nishitani, Euro Display 99 Proceedings, 53 (1999).

6. K. H. Kang, S. J. Lee, B. C. Song, M. S. Bang, S. E. Nam and H. J. Kim, J. Korean Phys. Soc., 44(6), 1552 (2004). 
7. E. Ibok and S. J. Garg, Electrochem. Soc., 140, 2927 (1993).

8. S. W. Lee and S. K. Joo, IEEE Electron Device Lett., 17, 160 (1997).

9. S. Y. Lee, Y. C. Jeon and S. K. Joo, Appl. Phys. Lett., 66(13), 1671 (1995).

10. J. S. Im and R. S. Sposili, Mat. Res. Bull., 2(3), 1671 (1995).

11. J. B. Lee, C. J. Lee and D. K. Choi, Jpn. J. Appl. Phys., 40, 6177 (2001).

12. A. R. Song, M. S. Thesis, Hongik University (2000).

13. R. Kakkad, J. Smith, W. S. Lau, S. J. Fonash and R. Kerns,
J. Appl. Phys., 65, 2069 (1989).

14. K. C. Park, I. H. Song, S. H. Jung, J. W. Park and M. K. Han, AM-LCD 2000, 147 (2000).

15. S. J. Lee, B. C. Song, S. H. Kim, S. K. Lee, M. S. Bang and S. E. Nam, J. Korean Phys. Soc., 47(2), 339, (2005).

16. A. Gat, L. Gerzberg, J. F. Gibbons, T. J. Magee, J. Peng and J. D. Hong, Appl. Phys. Lett., 33(9), 775 (1978).

17. Y. Kawasaki, T. Murakami, T. Kuroi, Y. Ohno and Y. Matsui, Mat. Chem. and Phys., 54, 17 (1998).

18. D. C. Montgomery and G. C. Runger, Applied statistics and probability for engineers, John Wiley \& Sons Inc, 505 (2002). 